Archive for

Organic Chemistry

Arkivoc 2020, part viii, 136-149

\title{
Hantzsch reaction with 6-aminouracil: Synthesis of novel tetrakis(6-aminouracil- 5-yl)methanes and bis(decahydropyrido[2,3-d:6,5-d']dipyrimidine-tetraones) linked to aliphatic or aromatic cores via ether-amide or ester-amide linkages
}

\author{
Amr M. Abdelmoniem, Amna M. Abdella, Ahmed H. M. Elwahy,* Ismail A. Abdelhamid* \\ Department of Chemistry, Faculty of Science, Cairo University,12613 Giza, A. R. Egypt \\ E-mail,ismailsshafy@yahoo.com
}

Received 09-05-2020

Accepted 11-29-2020

Published on line $12-14-2020$

\section{Abstract}

One-pot three-component cyclo-condensation reaction of bis(aldehydes) containing ether-amide or esteramide linkages with 6-aminouracil in boiling acetic acid afforded tetrakis(6-aminopyrimidine-2,4-diones) or bis(tetraoxodecahydropyrido[2,3-d:6,5- $d$ ']dipyrimidines) depending on the reaction conditions.

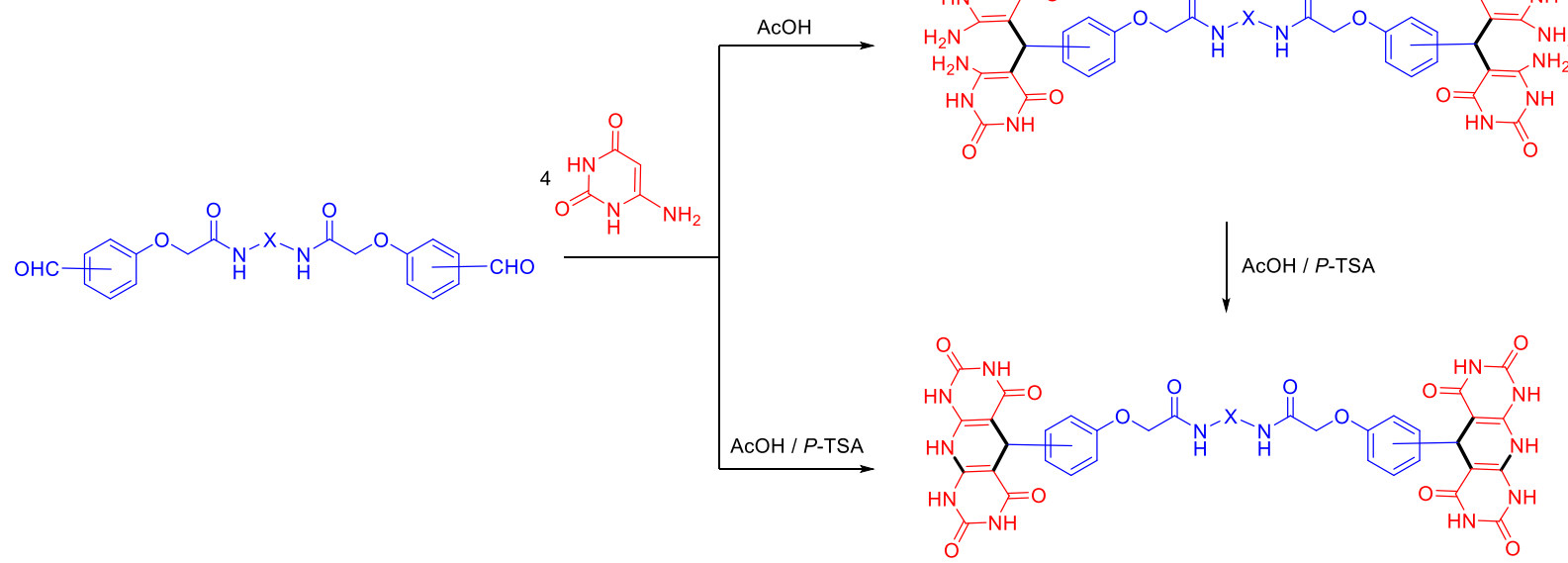

Keywords: Hantzsch reaction, bis(aldehydes), amide linkages, tetrakis(6-aminopyrimidine-2,4-diones), bis(tetraoxodecahydropyrido[2,3-d:6,5-d']dipyrimidines) 


\section{Introduction}

Hantzsch reaction is one of the most common routes for the synthesis of 1,4-dihydropyridines (1,4-DHPs) having therapeutic and pharmacological properties. ${ }^{1-19}$ In addition, Uracil is considered to be one of the major motifs present in the biopolymer RNA ${ }^{20,21}$ and it plays several roles in our life. ${ }^{22,23}$ The uracil scaffold and its derivatives exhibit a wide range of biological activities, including anticancer agents, ${ }^{24-26}$ antihypertensive agents, ${ }^{27}$ antiallergic compounds ${ }^{28}$ and antiviral agents. ${ }^{29-31}$ Structures of some FDA-approved uracil drugs are depicted in figure 1. Moreover, dipyrimidines exhibit a broad range of pharmacological properties, such as antimicrobial, ${ }^{32,33}$ antitumor, ${ }^{34}$ and antiviral. ${ }^{35,36}$ Furthermore, the multicomponent reactions (MCRs) provide an easy and rapid access to diversity of heterocycles as they have the advantages of atom-economy and selectivity. ${ }^{18,37-51}$<smiles>O=c1[nH]cc(F)c(=O)[nH]1</smiles>

Fluorouracil<smiles>O=c1cc(N(CCCl)CCCl)[nH]c(=O)[nH]1</smiles>

Uracil mustard<smiles>Nc1[nH]c(=O)[nH]c(=O)c1F</smiles>

Flucytosine Antifungal<smiles>Nc1ccn([C@H]2CC[C@@H](CO)O2)c(=O)n1</smiles>

Zalcitabine reverse transcriptase inhibitor against Human Immunodeficiency Virus Type 1 (HIV-1)

Figure 1. Some selected FDA-approved uracil drugs.

In connection with the importance of both 1,4-dihydropyridine and the dipyrimidine moiety and in continuation to our interest in enamine chemistry, ${ }^{52-56}$ the synthesis of bis(heterocycles) ${ }^{17,47-60}$ as well as the $C-C$ bond formation reactions, ${ }^{52,71-73}$ we report herein a highly efficient method for the synthesis of bis(pyrido[2,3-d:6,5-d']dipyrimidinetetraones) linked to aliphatic or aromatic core via ether-amide or esteramide linkages through the reaction of bis(aldehydes) with 6-aminouracil.

\section{Results and Discussion}

Firstly, the bis(2-(2-formylphenoxy)acetamide) 1a was prepared following our reported procedure via the reaction of the potassium salt of salicylaldehyde with $N, N^{\prime}$-(ethane-1,2-diyl)bis(2-chloroacetamide) in DMF. ${ }^{74}$ The reactivity of the bis(aldehyde) 1 a towards 6-aminouracil $\mathbf{2}$ was then investigated aiming at the synthesis of bis(decahydropyrido[2,3-d:6,5- $d^{\prime}$ ]dipyrimidin-5-yl)phenoxy)acetamide) 4a. Contrary to our expectation, the reaction did not yield compound $\mathbf{4 a}$, instead it gave the uncyclized tetrakis(6-aminopyrimidine-2,4-dione) 3a in $88 \%$ yield (Scheme 1). It worth mentioning that trials to cyclize similar systems in acetic acid at reflux were unsuccessful. ${ }^{75}$ On the other hand, heating a mixture of the bis(aldehyde) 1 a with the aminouracil 2 in acetic acid in the presence of $p$-TSA for $3 \mathrm{~h}$ afforded the target $4 \mathrm{a}$ as the sole product. Moreover, we have found that heating of compound $\mathbf{3 a}$ in acetic acid / $p$-TSA for $1 \mathrm{~h}$ afforded $4 \mathrm{a}$ directly in one step. 


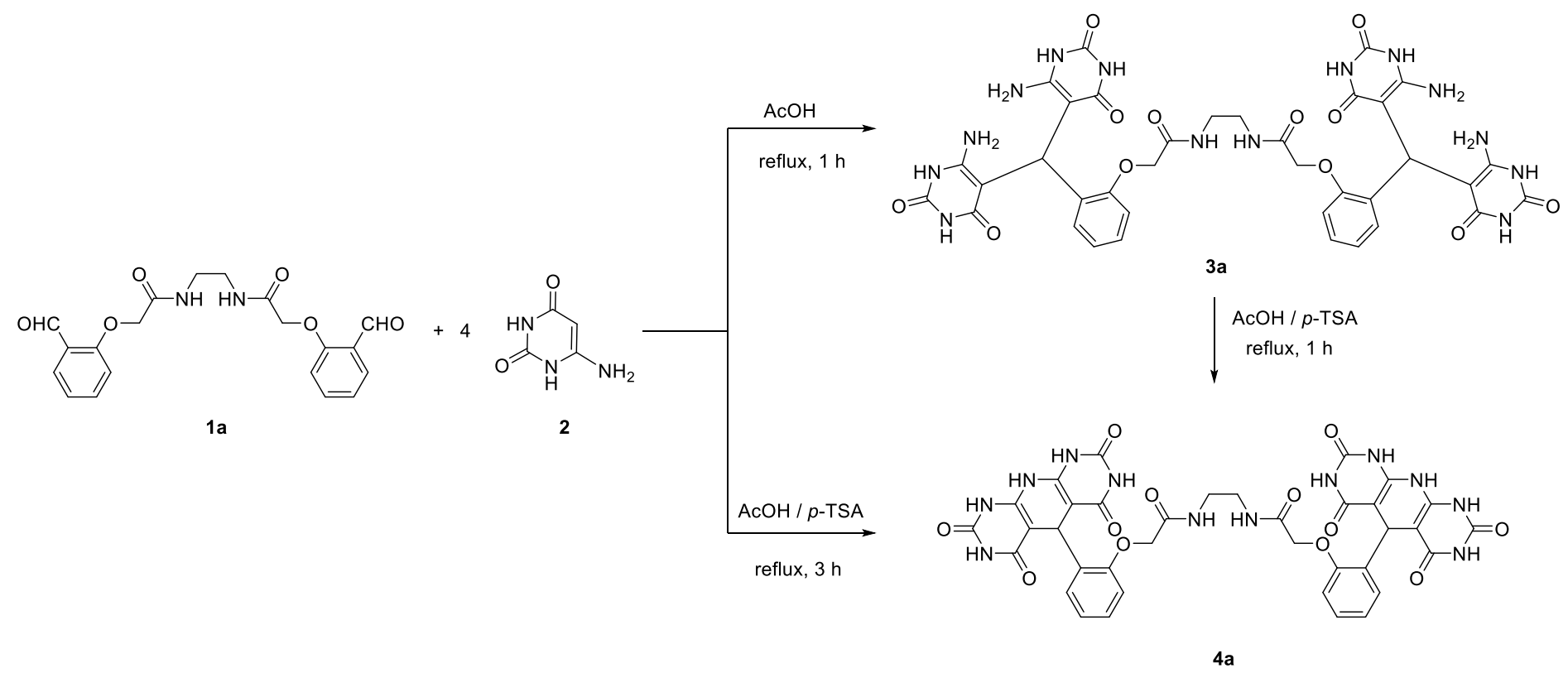

Scheme 1. Synthesis of tetrakis(6-aminopyrimidine-2,4-dione) 3a and bis(decahydropyrido[2,3-d:6,5$d^{\prime}$ ]dipyrimidin-5-yl)phenoxy)acetamide) 4a.

The structures of compounds $3 \mathbf{a}$ and 4 a were supported based on the different spectral tools. Thus, the ${ }^{1} \mathrm{H}$ NMR spectrum of compound 3a revealed a characteristic broad integrated by $4 \mathrm{H}$ at $\delta 3.30$ ppm for the two methylene linkage. It also showed two characteristic singlets at $\delta 4.31$ and $5.38 \mathrm{ppm}$ for the $-\mathrm{OCH}_{2} \mathrm{CO}-$ and the bridging $\mathrm{CH}$ protons, respectively. In addition, it exhibited four broad singlet signals characteristic for the $\mathrm{NH}_{2}$ and three $\mathrm{NH}$ groups at $\delta 6.74$ and $\delta 7.48,10.30$ and 10.42, respectively. It also featured aromatic protons at $\delta$ 6.83-7.15. On the other hand, the ${ }^{1} \mathrm{H}$ NMR spectrum of compound $4 \mathrm{a}$, indicated the disappearance of the characteristic broad signals at the range of 6-7 ppm for the amino groups. It also featured in addition to the signals of methylene groups, singlet signal at $\delta 5.13 \mathrm{ppm}$ for the pyridine-H5. It also indicated three different broad singlets at $\delta 8.41,10.64$ and 11.18 ppm corresponding to three different $\mathrm{NH}$ groups.

Stimulated by these noteworthy results, a series of bis(aldehydes) $\mathbf{1 b}$-f were prepared ${ }^{74}$ and used to ascertain the generality and the scope of the protocol. The reaction of 6-aminouracil $\mathbf{2}$ with the appropriate bis(aldehydes) $\mathbf{1 b}$-f in acetic acid afforded the corresponding tetrakis(uracil) $\mathbf{3 b}$-f (Scheme 2) . 


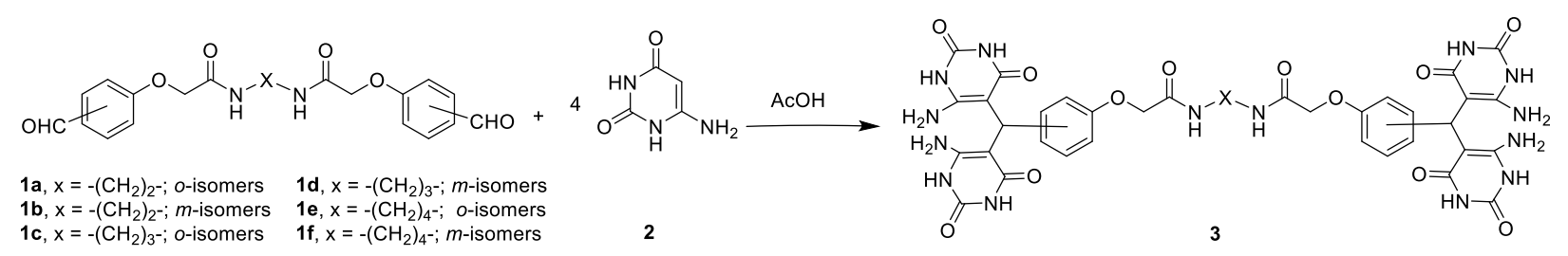

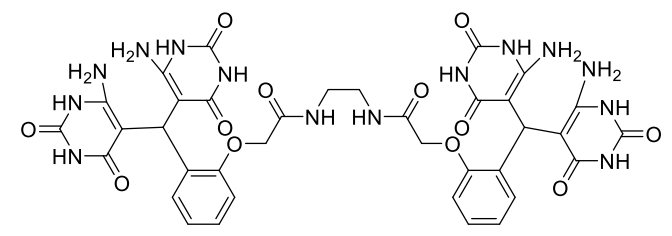

$3 a$

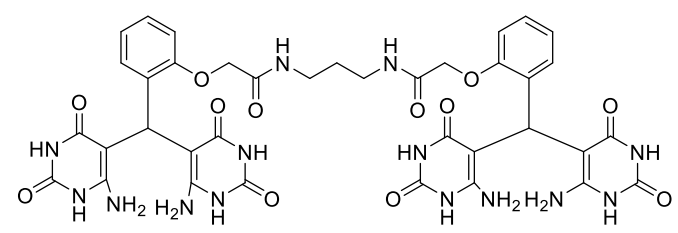

$3 c$

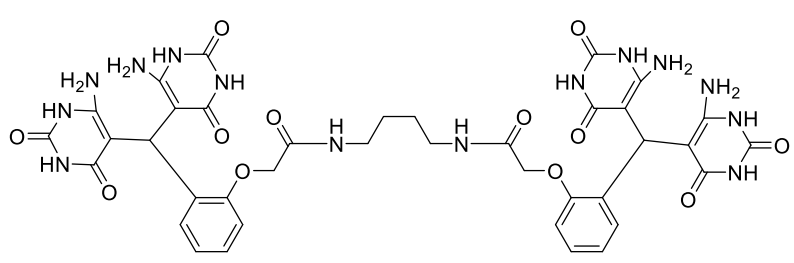

$3 e$
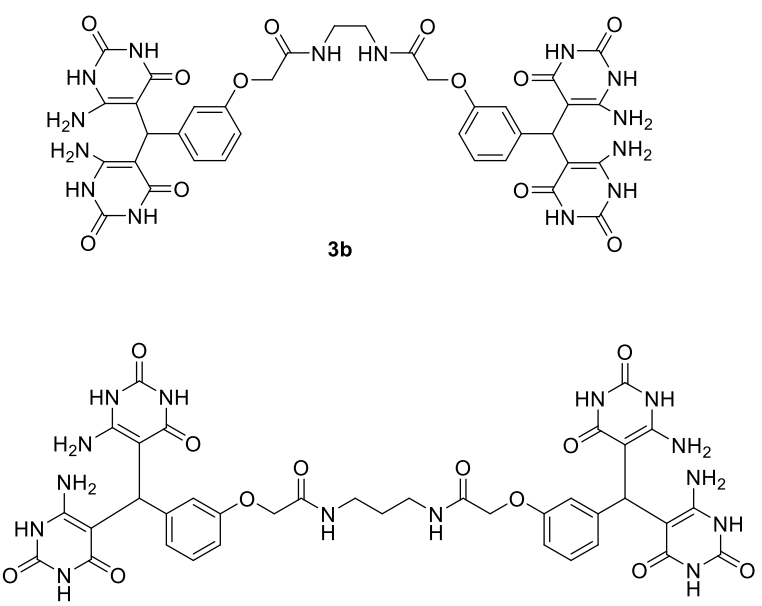

$3 d$

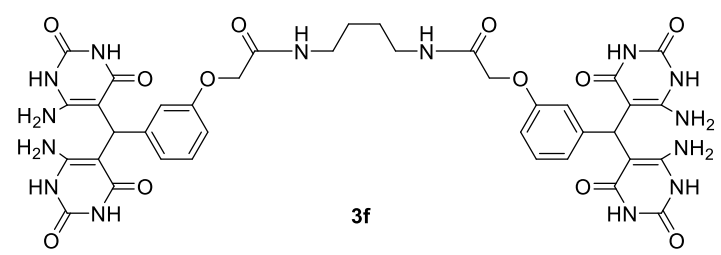

Scheme 2. Multicomponent synthesis of tetrakis(6-aminopyrimidine-2,4-dione) derivatives 3a-f.

However, the reaction of 6 -aminouracil 2 with the bis(aldehydes) 1a, 1c, 1e in acetic acid / p-TSA resulted in the formation of bis(decahydropyrido[2,3-d:6,5-d']dipyrimidin-5-yl)phenoxy)acetamides) 4a-c, respectively, in good yields (Scheme 3). The latter compounds were alternatively obtained in good yields, by heating the corresponding tetra-uracil $\mathbf{3 a}, \mathbf{3 c}$ and $\mathbf{3 e}$ in acetic acid containing $p$-TSA at reflux. 


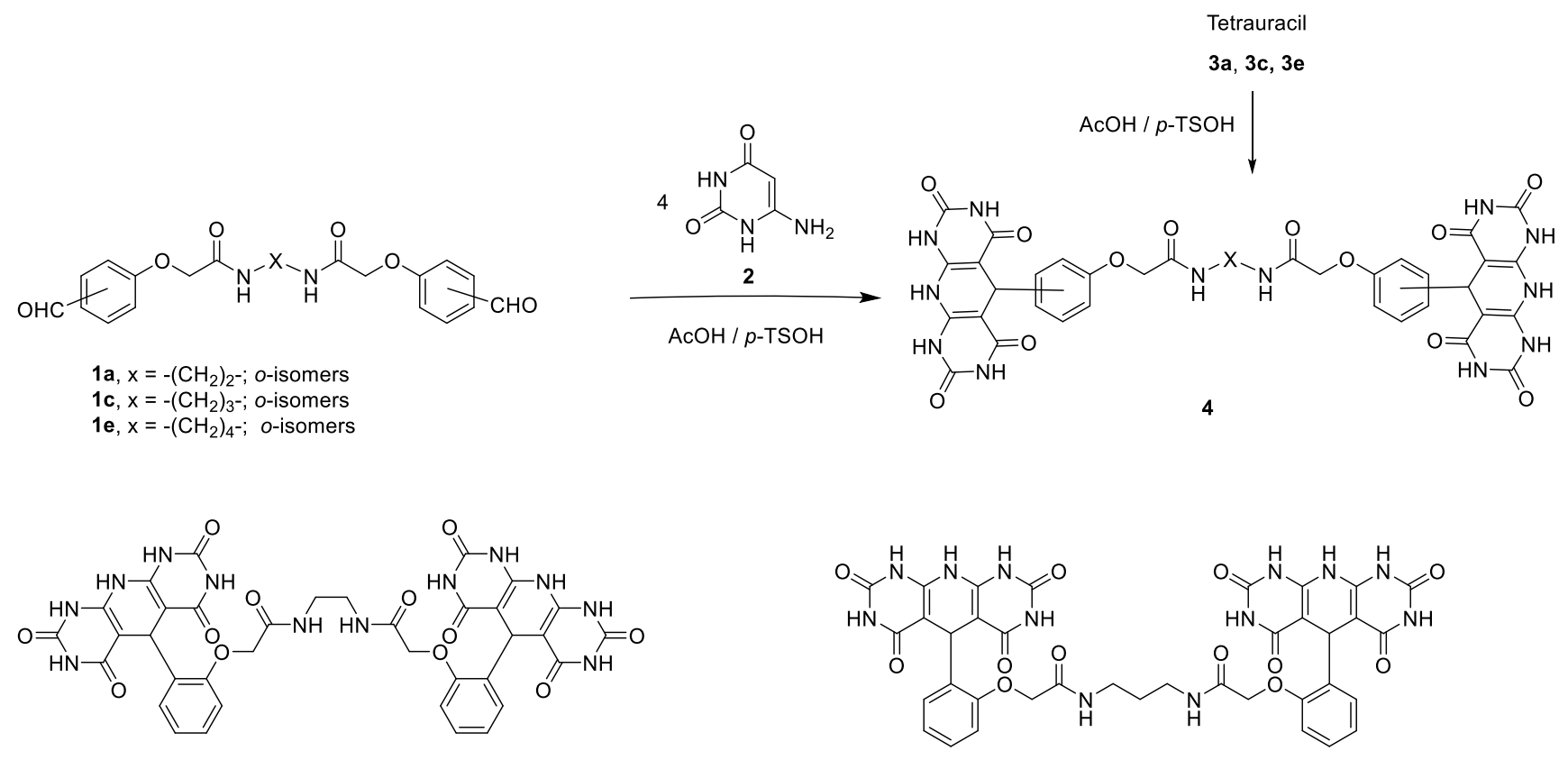

$4 a$

4b

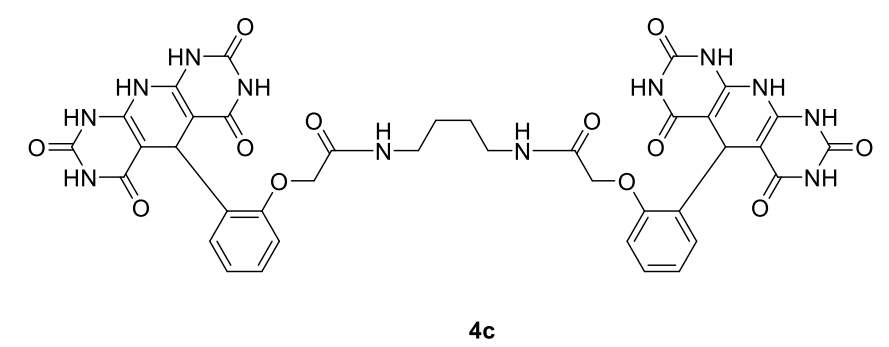

Scheme 3. Multicomponent synthesis of bis(decahydropyrido[2,3-d:6,5- $d$ ']dipyrimidin-5-yl)phenoxy)acetamide) derivatives $\mathbf{4 a - c .}$

The scope of the reaction was further extended towards aldehydes which are linked to the benzene core via ether-amide linkage. It has been found that the reaction of bis(aldehyde) $\mathbf{1 g}$ (aldehydic groups in meta position) with 6-aminouracil in acetic acid failed to give the target cyclized product pyridodipyrimidine even after prolonged heating in the presence of $p$-TSA (Scheme 4). The reaction gave instead the tetrakis(6aminopyrimidine-2,4-dione) $\mathbf{3 g}$. On the other hand, the reaction of the bis(aldehyde) $\mathbf{1 h}$ (aldehydic groups in ortho position) with 6-aminouracil $\mathbf{2}$ in acetic acid/p-TSA affords directly, the cyclic aromatized pyridodipyrimidine product $\mathbf{4 d}$ (Scheme 4). 


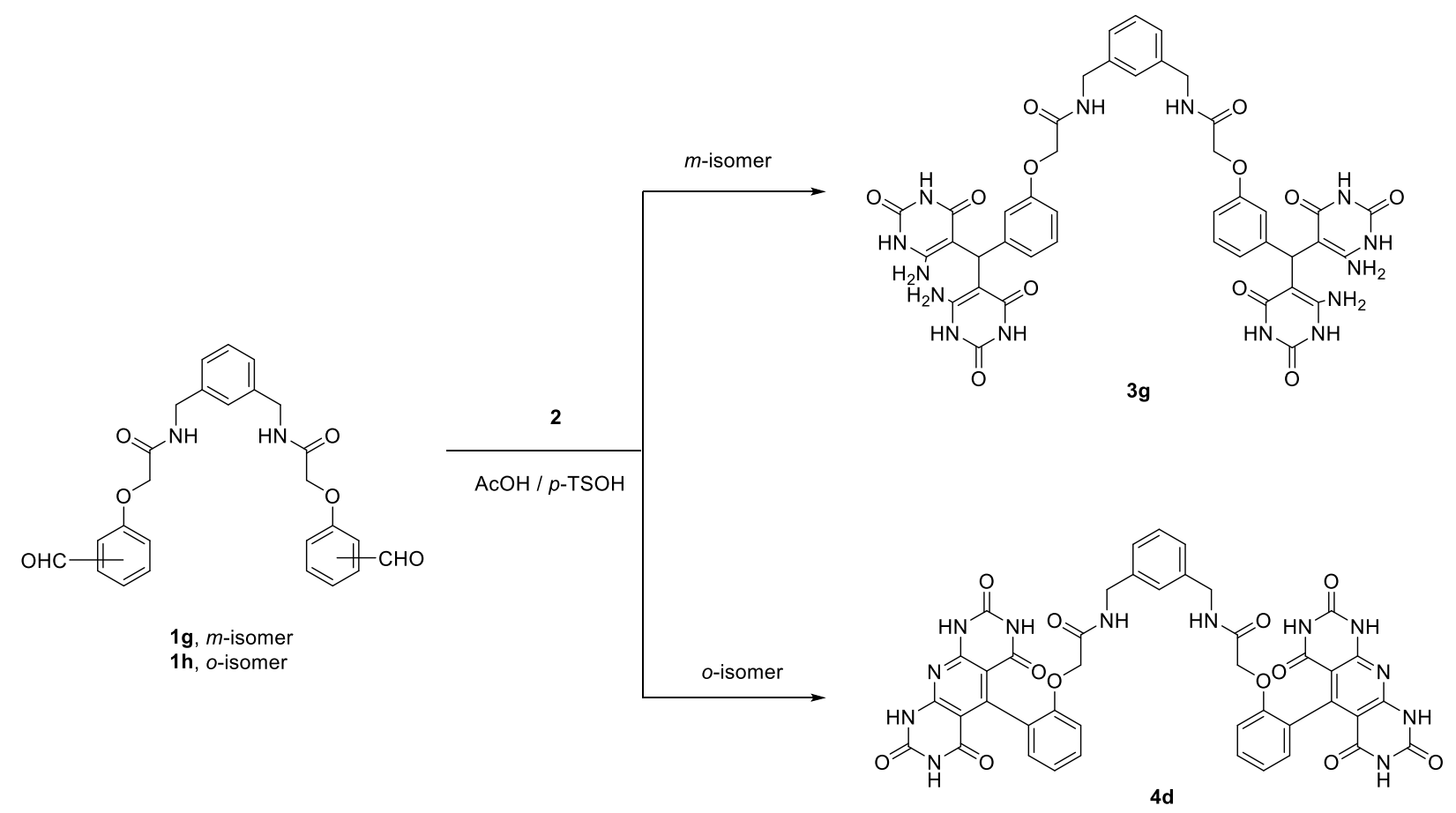

Scheme 4. Synthesis of tetrakis(6-aminopyrimidine-2,4-dione) $\mathbf{3 g}$ and bis(tetraoxo-octahydropyrido[2,3-d:6,5$d^{\prime}$ ']dipyrimidine) $\mathbf{4 d}$ which are linked to the benzene core via amide linkage.

Interestingly, the same methodology was also applied for the synthesis of the corresponding tetrakis(uracil) linked to ester-amide core linkage $\mathbf{8}$ via the direct reaction of the bis(aldehyde) $\mathbf{7}$ with four equivalents of 6 aminouracil 2 in acetic acid. Unfortunately, trials to cyclize $\mathbf{8}$ into bis(decahydropyrido[2,3-d:6,5$d^{\prime}$ ]dipyrimidin-5-yl)phenoxy)acetamide) 9 upon heating 8 in acetic acid / $p$-TSA were unsuccessful (Scheme 5). The aldehyde containing ester-amide linkage 7 was prepared via the reaction of the potassium salt of the $p$ formylbenzoic acid 6 with the corresponding bis(2-chloroacetamide) 5 in boiling DMF. ${ }^{74}$

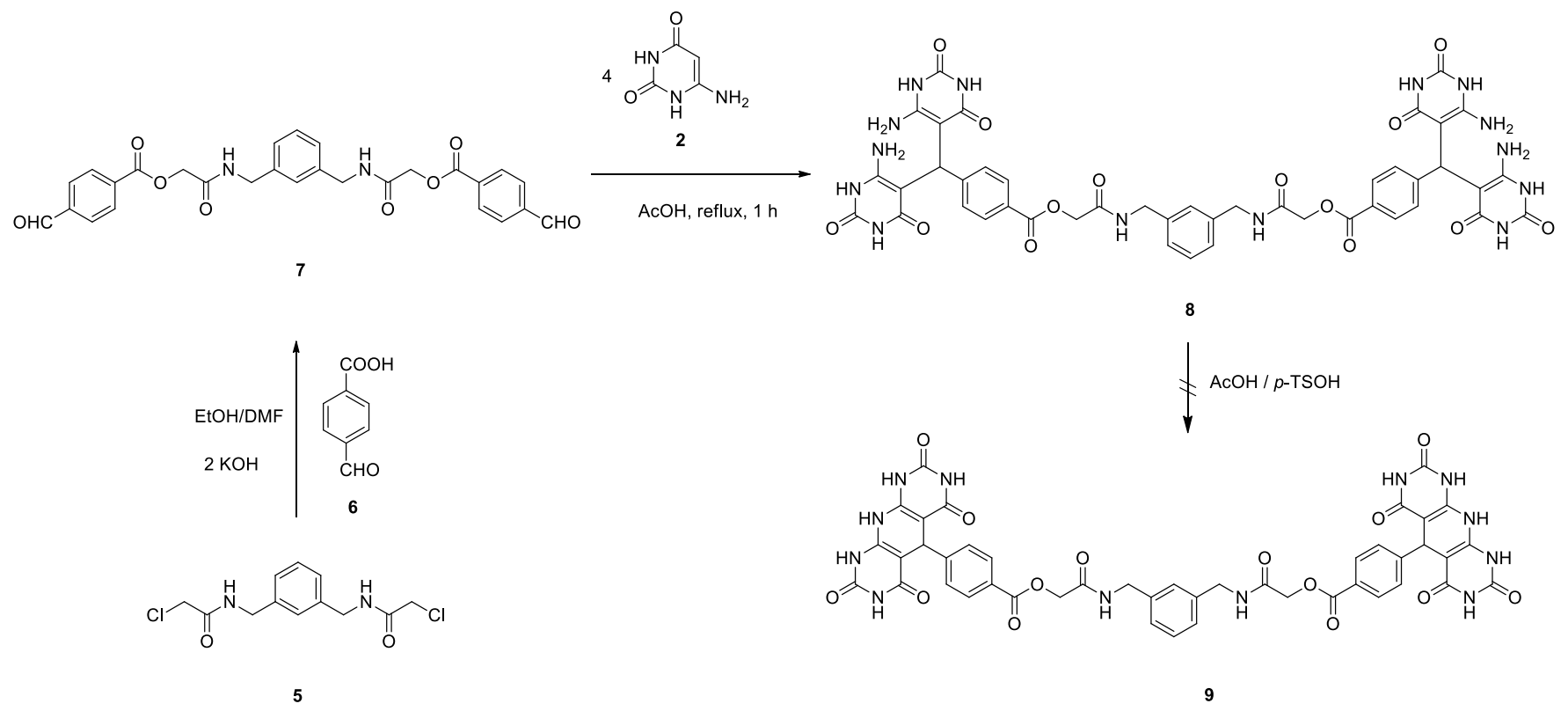

Scheme 5. Unsuccessful trial to obtain bis(decahydropyrido[2,3- $\left.d: 6,5-d^{\prime}\right]$ dipyrimidin-5-yl)phenoxy)-acetamide) 9. 
A proposed synthetic pathway for the reaction of bisaldehydes with 6-aminouracil is shown in scheme 5 . Nucleophilic addition of the enamine 6 -carbon of 6-aminouracil 2 to the two carbonyl centers of the bis(aldehydes) 1 affords the corresponding adducts 10. Subsequent elimination of water leads to the formation of the corresponding ene-imine intermediate 11. The Michael addition of another two moles of 6aminouracil $\mathbf{2}$ to the intermediate $\mathbf{1 1}$ affords the products 3. Subsequent removal of ammonia using $p$-TSA led to the formation of $\mathbf{4}$ (Scheme 6).

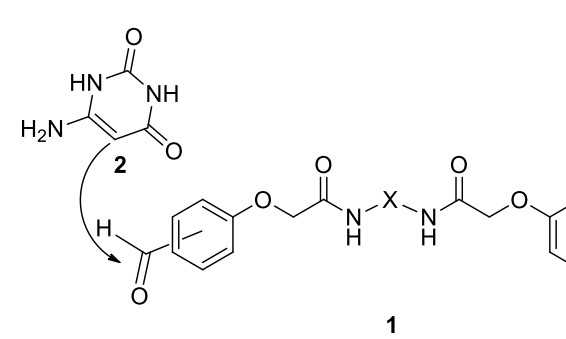

1

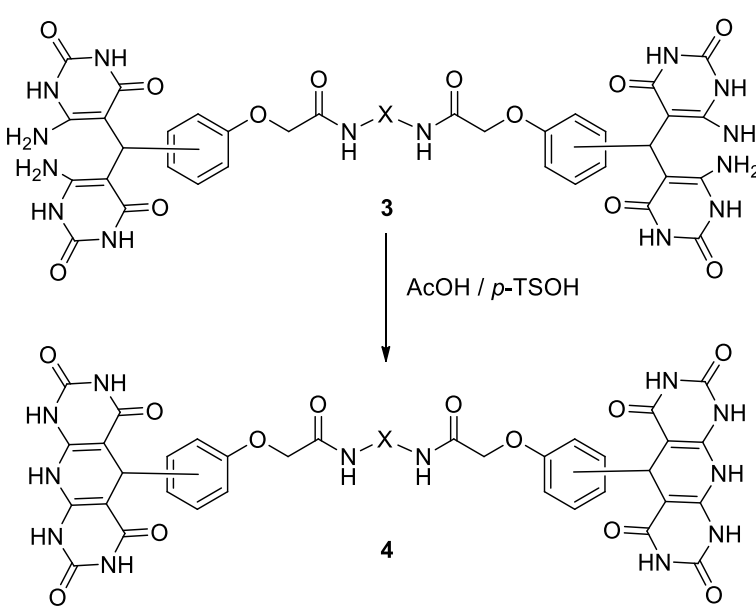

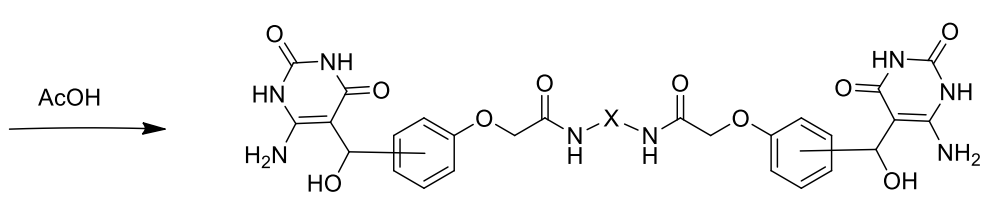

10

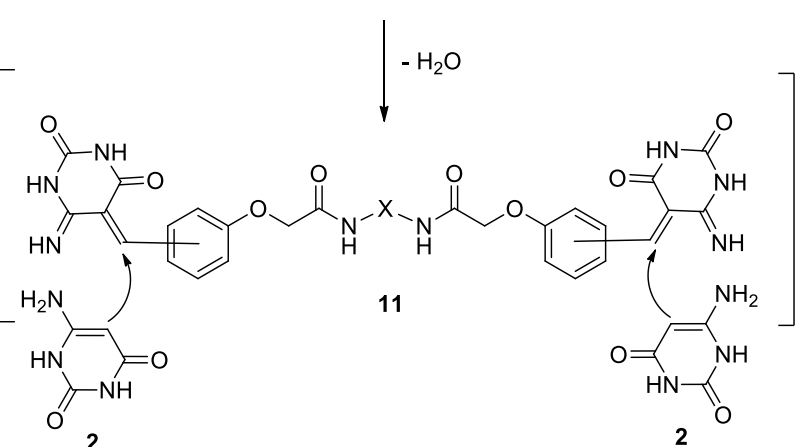

Scheme 6. A proposed pathway for the synthesis of compounds $\mathbf{3}$ and $\mathbf{4}$.

\section{Conclusions}

We developed a synthetic approach for the synthesis of novel tetrakis(6-aminopyrimidine-2,4-diones) or bis(tetraoxodecahydropyrido[2,3-d:6,5-d']dipyrimidines) via one-pot three-component cyclo-condensation reaction of bis(aldehydes) containing ether-amide or ester-amide linkages with 6-aminouracil. The reaction products was found to be dependent on the reaction conditions. It is expected that the novel structures prepared in this paper would be useful in the field of medicinal and synthetic organic chemistry.

\section{Experimental Section}

General. Melting points were measured with a Stuart melting point apparatus and are uncorrected. The IR spectra were recorded using a FTIR Bruker-vector 22 spectrophotometer as $\mathrm{KBr}$ pellets. The ${ }^{1} \mathrm{H}$ and ${ }^{13} \mathrm{C}$ NMR spectra were recorded in DMSO- $d_{6}$ as solvent on Varian Gemini NMR spectrometer at $400 \mathrm{MHz}$ and $100 \mathrm{MHz}$, respectively, using TMS as internal standard. Chemical shifts are reported as $\delta$ values in ppm. Mass spectra 
were recorded with a Shimadzu GCMS-QP-1000 EX mass spectrometer in El (70 eV) model. Elemental analyses were performed on a Perkin-Elmer 240 micoanalyser at the Micro analytical Center of Cairo University.

General procedure for the synthesis of $\mathbf{3 a - 3 g}$ and $\mathbf{8}$. A solution of each of bisaldehydes $\mathbf{1 a - 1 g}$ and 7 (1 $\mathrm{mmol})$ and 6-aminouracil $(4 \mathrm{mmol})$ in acetic acid $(3 \mathrm{ml})$ was heated at reflux for $1 \mathrm{~h}$. The solid obtained was collected and crystallized from DMF/EtOH to give compounds $3 a-g$ and 8.

$N, N^{\prime}$-(Ethane-1,2-diyl)bis(2-(2-(bis(6-amino-2,4-dioxo-1,2,3,4-tetrahydropyrimidin-5-yl)methyl)phenoxy)acetamide) (3a). Pale yellow powder (88\%). mp 270-272 ${ }^{\circ} \mathrm{C}$. IR (KBr): v 3344, $3155\left(\mathrm{NH}\right.$ and $\left.\mathrm{NH}_{2}\right), 1708(\mathrm{CO})$. ${ }^{1} \mathrm{H}$ NMR (300 MHz, DMSO-d $\left.d_{6}\right): \delta 3.30\left(\mathrm{br}, 4 \mathrm{H}, \mathrm{CH}_{2} \mathrm{~N}\right), 4.31\left(\mathrm{~s}, 4 \mathrm{H}, \mathrm{CH}_{2} \mathrm{O}\right), 5.38(\mathrm{~s}, 2 \mathrm{H}, \mathrm{CH}), 6.47\left(\mathrm{br}, 8 \mathrm{H}, \mathrm{NH}_{2}\right)$, 6.83-7.15 (m, 8H, Ar-H), $7.48(\mathrm{br}, 2 \mathrm{H}, \mathrm{NH}), 10.30(\mathrm{br}, 4 \mathrm{H}, \mathrm{NH}), 10.42(\mathrm{br}, 4 \mathrm{H}, \mathrm{NH}) .{ }^{13} \mathrm{C} \mathrm{NMR}(100 \mathrm{MHz}, \mathrm{DMSO}-$ $\left.d_{6}\right): \delta 29.7,36.3,68.1,112.8,121.3,127.2,128.4,129.2,150.2,153.9,156.0,162.8,165.6,168.4$. MS (El, 70 eV): $m / z$ (\%) $856\left[\mathrm{M}^{+}\right]$. Anal. Calcd for $\mathrm{C}_{36} \mathrm{H}_{36} \mathrm{~N}_{14} \mathrm{O}_{12}: \mathrm{C}, 50.47 ; \mathrm{H}, 4.24 ; \mathrm{N}, 22.89$ found $\mathrm{C}, 50.73 ; \mathrm{H}, 4.43 ; \mathrm{N}$, 23.13.

$N, N^{\prime}$-(Ethane-1,2-diyl)bis(2-(3-(bis(6-amino-2,4-dioxo-1,2,3,4-tetrahydropyrimidin-5-yl)methyl)phenoxy)acetamide) (3b). Pale yellow powder (85\%). mp 288-290 ${ }^{\circ} \mathrm{C}$. IR (KBr): v 3325, $3147(\mathrm{NH} \text { and NH})^{2}, 1712$ (CO). ${ }^{1} \mathrm{H}$ NMR (400 MHz, DMSO-d $\left.)_{6}\right): \delta 3.24\left(\mathrm{br}, 4 \mathrm{H}, \mathrm{CH}_{2} \mathrm{~N}\right), 4.36\left(\mathrm{~s}, 4 \mathrm{H}, \mathrm{CH}_{2} \mathrm{O}\right), 5.30(\mathrm{~s}, 2 \mathrm{H}, \mathrm{CH}), 6.70-7.15(\mathrm{~m}, 16 \mathrm{H}$,

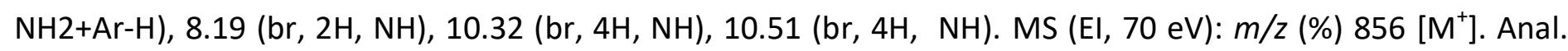
Calcd for $\mathrm{C}_{36} \mathrm{H}_{36} \mathrm{~N}_{14} \mathrm{O}_{12}$ : C, 50.47; H, 4.24; N, 22.89 found $\mathrm{C}, 50.69 ; \mathrm{H}, 4.07 ; \mathrm{N}, 23.14$.

N,N'-(Propane-1,3-diyl)bis(2-(2-(bis(6-amino-2,4-dioxo-1,2,3,4-tetrahydropyrimidin-5-yl)methyl)phenoxy)acetamide) (3c). Pale yellow powder (90\%). mp 242-244 ${ }^{\circ} \mathrm{C}$. IR (KBr): v 3348, $3171\left(\mathrm{NH}\right.$ and $\left.\mathrm{NH}_{2}\right), 2978(\mathrm{NH})$, $2850\left(\mathrm{NH}_{2}\right), 1712$ (CO). ${ }^{1} \mathrm{H}$ NMR (300 MHz, DMSO-d $): \delta 1.60-1.62(\mathrm{~m}, 2 \mathrm{H}, \mathrm{CH} 2), 3.14-3.17\left(\mathrm{~m}, 4 \mathrm{H}, \mathrm{CH}_{2} \mathrm{~N}\right), 4.34$ (s, 4H, CH $\left.\mathrm{CH}_{2} \mathrm{O}\right), 5.34(\mathrm{~s}, 2 \mathrm{H}, \mathrm{CH}), 6.50\left(\mathrm{br}, 8 \mathrm{H}, \mathrm{NH}_{2}\right), 6.81-7.11(\mathrm{~m}, 8 \mathrm{H}, \mathrm{Ar}-\mathrm{H}), 7.14(\mathrm{br}, 2 \mathrm{H}, \mathrm{NH}), 10.45(\mathrm{br}, 8 \mathrm{H}, \mathrm{NH})$. ${ }^{13} \mathrm{C}$ NMR $\left(75 \mathrm{MHz}\right.$, DMSO- $d_{6}$ ): $\delta$ 20.9, 29.2, 36.1, 67.2, 86.5, 111.8, 120.6, 120.7, 126.6, 128.6, 149.6, 153.4, 155.3, 165.0, 167.5. MS (El, $70 \mathrm{eV}): \mathrm{m} / \mathrm{z}(\%) 870\left[\mathrm{M}^{+}\right]$. Anal. Calcd for $\mathrm{C}_{37} \mathrm{H}_{38} \mathrm{~N}_{14} \mathrm{O}_{12}: \mathrm{C}, 51.03 ; \mathrm{H}, 4.40 ; \mathrm{N}, 22.52$ found C, 51.31; H, 4.66; N, 22.25.

$N, N^{\prime}$-(Propane-1,3-diyl)bis(2-(3-(bis(6-amino-2,4-dioxo-1,2,3,4-tetrahydropyrimidin-5-yl)methyl)phenoxy)acetamide) (3d). Pale yellow powder (87\%). $\mathrm{mp}>300{ }^{\circ} \mathrm{C}$. IR (KBr): v 3325, $3178\left(\mathrm{NH}\right.$ and $\left.\mathrm{NH}_{2}\right), 1724(\mathrm{CO}) .{ }^{1} \mathrm{H}$ NMR (300 MHz, DMSO- $\left.d_{6}\right): \delta$ 1.57-1.64 (m, 2H, CH2), 3.11-3.13 (m, 4H, $\left.\mathrm{CH}_{2} \mathrm{~N}\right), 4.37\left(\mathrm{~s}, 4 \mathrm{H}, \mathrm{CH}_{2} \mathrm{O}\right), 5.30(\mathrm{~s}, 2 \mathrm{H}$, $\mathrm{CH}), 6.69\left(\mathrm{br}, 8 \mathrm{H}, \mathrm{NH}_{2}\right), 6.72-7.13(\mathrm{~m}, 8 \mathrm{H}, \mathrm{Ar}-\mathrm{H}), 8.08(\mathrm{br}, 2 \mathrm{H}, \mathrm{NH}), 10.28(\mathrm{br}, 4 \mathrm{H}, \mathrm{NH}), 10.47(\mathrm{br}, 4 \mathrm{H}, \mathrm{NH}) .{ }^{13} \mathrm{C}$ NMR $\left(100 \mathrm{MHz}\right.$, DMSO- $\left.d_{6}\right): \delta$ 29.7, 32.9, 36.2, 67.5, 110.8, 114.6, 120.4, 129.1, 138.0, 142.0, 150.2, 158.0, 167.8, 168.2, 172.7. MS (El, $70 \mathrm{eV}): \mathrm{m} / \mathrm{z}(\%) 870\left[\mathrm{M}^{+}\right]$. Anal. Calcd for $\mathrm{C}_{37} \mathrm{H}_{38} \mathrm{~N}_{14} \mathrm{O}_{12}$ : .C, 51.03; $\mathrm{H}, 4.40 ; \mathrm{N}, 22.52$ found C, 51.32; H, 4.60; N, 22.20.

N,N'-(Butane-1,4-diyl)bis(2-(2-(bis(6-amino-2,4-dioxo-1,2,3,4-tetrahydropyrimidin-5-yl)methyl)phenoxy)acetamide) (3e). Pale yellow powder (91\%). mp 242-244 ${ }^{\circ} \mathrm{C}$. IR (KBr): v 3429, 3367, $3179\left(\mathrm{NH}_{\text {and }} \mathrm{NH}_{2}\right), 1724$ (CO). ${ }^{1} \mathrm{H}$ NMR (300 MHz, DMSO-d $\left.d_{6}\right):$ ): $\delta 1.42\left(\mathrm{br}, 4 \mathrm{H}, \mathrm{CH}_{2}\right)$, ), $3.16\left(\mathrm{br}, 4 \mathrm{H}, \mathrm{CH}_{2} \mathrm{~N}\right), 4.34\left(\mathrm{~s}, 4 \mathrm{H}, \mathrm{CH}_{2} \mathrm{O}\right)$, ), $5.34(\mathrm{~s}$, $\left.2 \mathrm{H}, \mathrm{CH}), 6.46\left(\mathrm{br}, 8 \mathrm{H}, \mathrm{NH}_{2}\right), 6.83-7.11(\mathrm{~m}, 8 \mathrm{H}, \mathrm{Ar}-\mathrm{H}), 7.12(\mathrm{br}, 2 \mathrm{H}, \mathrm{NH}), 10.33(\mathrm{br}, 4 \mathrm{H}, \mathrm{NH}), 10.42(\mathrm{br}, 4 \mathrm{H}, \mathrm{NH})\right)$. MS (El, $70 \mathrm{eV}): m / z(\%) 884\left[\mathrm{M}^{+}\right.$]. Anal. Calcd for $\mathrm{C}_{38} \mathrm{H}_{40} \mathrm{~N}_{14} \mathrm{O}_{12}: \mathrm{C}, 51.58 ; \mathrm{H}, 4.56 ; \mathrm{N}, 22.16$ found $\mathrm{C}, 51.84 ; \mathrm{H}$, 4.75; N, 22.45.

N,N'-(Butane-1,4-diyl)bis(2-(3-(bis(6-amino-2,4-dioxo-1,2,3,4-tetrahydropyrimidin-5-yl)methyl)phenoxy)acetamide)(3f). Pale yellow powder (88\%). mp 284-286 ${ }^{\circ} \mathrm{C}$. IR (KBr): v 3379, $3125\left(\mathrm{NH}\right.$ and $\left.\mathrm{NH}_{2}\right), 1708$ (CO). ${ }^{1} \mathrm{H}$ NMR (300 MHz, DMSO-d $)_{6}$ : ): $\delta 1.41\left(\mathrm{br}, 4 \mathrm{H}, \mathrm{CH}_{2}\right), 3.12\left(\mathrm{br}, 4 \mathrm{H}, \mathrm{CH}_{2} \mathrm{~N}\right), 4.35\left(\mathrm{~s}, 4 \mathrm{H}, \mathrm{CH}_{2} \mathrm{O}\right), 5.29(\mathrm{~s}, 2 \mathrm{H}, \mathrm{CH}), 6.68$ (br, 8H, NH $\mathrm{NH}_{2}$, 6.70-7.13 (m, 8H, Ar-H), 8.03 (br, 2H, NH), $10.28(\mathrm{br}, 4 \mathrm{H}, \mathrm{NH}), 10.47$ (br, 4H, NH). ${ }^{13} \mathrm{C} \mathrm{NMR} \mathrm{(100}$ $\left.\mathrm{MHz}, \mathrm{DMSO}-d_{6}\right): \delta 27.1,32.8,38.5,67.4,110.7,114.5,120.3,129.0,142.0,150.2,158.0,158.9,165.1,168.0$, 
172.8. MS (El, $70 \mathrm{eV}): \mathrm{m} / z$ (\%) $884\left[\mathrm{M}^{+}\right]$. Anal. Calcd for $\mathrm{C}_{38} \mathrm{H}_{40} \mathrm{~N}_{14} \mathrm{O}_{12}: \mathrm{C}, 51.58 ; \mathrm{H}, 4.56 ; \mathrm{N}, 22.16$ found C, $51.35 ; \mathrm{H}, 4.35 ; \mathrm{N}, 22.42$.

N,N'-(1,3-Phenylenebis(methylene))bis(2-(3-(bis(6-amino-2,4-dioxo-1,2,3,4-tetrahydropyrimidin-5-yl)methyl)phenoxy)acetamide) (3g). Pale yellow powder (89\%). mp 296-298 ${ }^{\circ} \mathrm{C}$. IR (KBr): v 3356, 3152 (NH and $\mathrm{NH}_{2}$ ), 1716 (CO). ${ }^{1} \mathrm{H}$ NMR (300 MHz, DMSO-d $)_{6}$ : 4.30-4.32 (d, J = 5.7 Hz, 4H, CH$\left.{ }_{2} \mathrm{~N}\right), 4.44\left(\mathrm{~s}, 4 \mathrm{H}, \mathrm{CH}_{2} \mathrm{O}\right), 5.30(\mathrm{~s}$, $2 \mathrm{H}, \mathrm{CH}), 6.70\left(\mathrm{br}, 8 \mathrm{H}, \mathrm{NH}_{2}\right), 6.73-7.15(\mathrm{~m}, 12 \mathrm{H}, \mathrm{Ar}-\mathrm{H}), 8.59-8.62(\mathrm{t}, J=6.6 \mathrm{~Hz}, 2 \mathrm{H}, \mathrm{NH}), 10.29(\mathrm{br}, 4 \mathrm{H}, \mathrm{NH}), 10.47$ (br, 4H, NH). ${ }^{13} \mathrm{C}$ NMR (100 MHz, DMSO-d $\left.d_{6}\right): \delta 33.1,42.2,67.4,110.7,114.6,120.3,126.2,126.8,128.7,129.0$, 139.8, 139.9, 142.0, 150.2, 158.0, 167.9, 168.2, 172.8. MS (El, 70 eV): m/z (\%) 932[M $\left.{ }^{+}\right]$. Anal. Calcd for $\mathrm{C}_{42} \mathrm{H}_{40} \mathrm{~N}_{14} \mathrm{O}_{12}$ : C, 54.08; $\mathrm{H}, 4.32 ; \mathrm{N}, 21.02$ found $\mathrm{C}, 53.89 ; \mathrm{H}, 4.12 ; \mathrm{N}, 21.22$.

((1,3-Phenylenebis(methylene))bis(azanediyl))bis(2-oxoethane-2,1-diyl)bis(4-(bis(6-amino-2,4-dioxo-1,2,3,4tetrahydropyrimidin-5-yl)methyl)benzoate) (8). Pale yellow powder (86\%). mp 294-296 ${ }^{\circ} \mathrm{C} . \mathrm{IR}(\mathrm{KBr}): \mathrm{v} 3368$, $3151\left(\mathrm{NH}\right.$ and $\mathrm{NH}_{2}$ ), 1713 (CO), 1624 (CO). ${ }^{1} \mathrm{H}$ NMR (300 MHz, DMSO-d $)$ : 4.30-4.32 (d, J = 5.4 Hz, 4H, CH $\mathrm{CH}_{2}$ ), $4.75\left(\mathrm{~s}, 4 \mathrm{H}, \mathrm{CH}_{2} \mathrm{O}\right), 5.36(\mathrm{~s}, 2 \mathrm{H}, \mathrm{CH}), 6.71\left(\mathrm{br}, 8 \mathrm{H}, \mathrm{NH}_{2}\right), 7.14-7.16(\mathrm{~m}, 4 \mathrm{H}, \mathrm{Ar}-\mathrm{H}), 7.23-7.26(\mathrm{~d}, \mathrm{~J}=8.7 \mathrm{~Hz}, 4 \mathrm{H}, \mathrm{Ar}-$ $\mathrm{H}), 7.87-7.90(\mathrm{~d}, J=8.7 \mathrm{~Hz}, 4 \mathrm{H}, \mathrm{Ar}-\mathrm{H}), 8.60-8.62(\mathrm{t}, J=6 \mathrm{~Hz}, 2 \mathrm{H}, \mathrm{NH}), 10.33(\mathrm{br}, 4 \mathrm{H}, \mathrm{NH}), 10.52(\mathrm{br}, 4 \mathrm{H}, \mathrm{NH}) .{ }^{13} \mathrm{C}$ NMR $\left.(100 \mathrm{MHz} \text {, DMSO-d })_{6}\right): \delta 33.3,42.3,63.3,126.1,126.6,127.4,128.8,129.6,130.0,130.7,139.7,146.7$, 150.2, 164.7, 165.9, 167.3, 172.5. MS (El, $70 \mathrm{eV}): \mathrm{m} / z$ (\%) $988\left[\mathrm{M}^{+}\right]$. Anal. Calcd for $\mathrm{C}_{44} \mathrm{H}_{40} \mathrm{~N}_{14} \mathrm{O}_{14}: \mathrm{C}, 53.44 ; \mathrm{H}$, 4.08; N, 19.83 found C, 53.70; H, 3.86; N, 19.64.

General procedure for the synthesis of $\mathbf{4 a - d}$. A solution of each of bisaldehydes (1a, $1 \mathrm{c}, \mathbf{1 e}$ and $\mathbf{1 h})(1 \mathrm{mmol})$ and 6-aminouracil $(4 \mathrm{mmol})$ in acetic acid $(3 \mathrm{ml})$ in the presence of $p$-TSA was heated at reflux for $1 \mathrm{~h}$. The solid obtained was collected and crystallized from DMF/EtOH to give compounds $4 a-d$.

N,N'-(Ethane-1,2-diyl)bis(2-(2-(2,4,6,8-tetraoxo-1,2,3,4,5,6,7,8,9,10-decahydropyrido[2,3-d:6,5-d']-

dipyrimidin-5-yl)phenoxy)acetamide) (4a). Pale yellow powder (93\%). mp >300 ${ }^{\circ} \mathrm{C}$. IR ( $\left.\mathrm{KBr}\right): \mathrm{v} 3070(\mathrm{NH}), 1690$ (CO). ${ }^{1} \mathrm{H}$ NMR (300 MHz, DMSO-d $d_{6}$ ): 3.24 (br, 4H, $\mathrm{CH}_{2} \mathrm{~N}$ ), $4.46\left(\mathrm{~s}, 4 \mathrm{H}, \mathrm{CH}_{2} \mathrm{O}\right), 5.13$ (s, 2H, Pyridine-H), 6.72-7.10 $(\mathrm{m}, 10 \mathrm{H}, \mathrm{Ar}-\mathrm{H}+\mathrm{NH}), 8.41(\mathrm{br}, 2 \mathrm{H}, \mathrm{NHCO}), 10.64(\mathrm{br}, 4 \mathrm{H}, \mathrm{NH}), 11.18(\mathrm{br}, 4 \mathrm{H}, \mathrm{NH}) .{ }^{13} \mathrm{C} \mathrm{NMR}\left(100 \mathrm{MHz}, \mathrm{DMSO}-d_{6}\right)$ : $\delta$ 26.3, 38.3, 67.5, 90.2, 111.5, 121.5, 127.6, 130.0, 136.1, 144.1, 150.2, 154.7, 163.1, 170.0. MS (El, $70 \mathrm{eV})$ : $\mathrm{m} / \mathrm{z}(\%) 822\left[\mathrm{M}^{+}\right]$. Anal. Calcd for $\mathrm{C}_{36} \mathrm{H}_{30} \mathrm{~N}_{12} \mathrm{O}_{12}: \mathrm{C}, 52.56 ; \mathrm{H}, 3.68 ; \mathrm{N}, 20.43$ found $\mathrm{C}, 52.77 ; \mathrm{H}, 3.44 ; \mathrm{N}, 20.61$.

N,N'-(Propane-1,3-diyl)bis(2-(2-(2,4,6,8-tetraoxo-1,2,3,4,5,6,7,8,9,10-decahydropyrido[2,3-d:6,5-d']-

dipyrimidin-5-yl)phenoxy)acetamide) (4b). Pale yellow powder (91\%). $\mathrm{mp}>300{ }^{\circ} \mathrm{C}$. IR (KBr): v 3035 (br, $\left.\mathrm{NH}\right)$, 1690 (CO). ${ }^{1} \mathrm{H}$ NMR (300 MHz, DMSO-d $\left.d_{6}\right):$ 1.50-1.60 (m, $\left.2 \mathrm{H}, \mathrm{CH} 2\right), 3.04-3.10\left(\mathrm{~m}, 4 \mathrm{H}, \mathrm{CH}_{2} \mathrm{~N}\right), 4.49\left(\mathrm{~s}, 4 \mathrm{H}, \mathrm{CH}_{2} \mathrm{O}\right)$, 5.14 (s, 2H, Pyridine-H), 6.72-7.12 (m, 10H, Ar-H+NH), 8.34 (br, 4H, NHCO), 10.65 (br, 4H, NH), $11.18(\mathrm{br}, 4 \mathrm{H}$, $\mathrm{NH}) .{ }^{13} \mathrm{C}$ NMR $\left(100 \mathrm{MHz}\right.$, DMSO- $\left.d_{6}\right): \delta 29.2,36.6,38.3,67.6,90.2,111.5,121.6,127.7,130.2,135.8,144.3$, 150.3, 154.6, 163.2, 168.7. MS (El, $70 \mathrm{eV}): \mathrm{m} / \mathrm{z}(\%) 836\left[\mathrm{M}^{+}\right]$. Anal. Calcd for $\mathrm{C}_{37} \mathrm{H}_{32} \mathrm{~N}_{12} \mathrm{O}_{12}: \mathrm{C}, 53.11 ; \mathrm{H}, 3.85 ; \mathrm{N}$, 20.09 found $C, 52.89 ; \mathrm{H}, 4.10 ; \mathrm{N}, 20.30$.

N,N'-(Butane-1,4-diyl)bis(2-(2-(2,4,6,8-tetraoxo-1,2,3,4,5,6,7,8,9,10-decahydropyrido[2,3-d:6,5-d']-

dipyrimidin-5-yl)phenoxy)acetamide) (4c). Pale yellow powder (94\%). mp 294-296 ${ }^{\circ} \mathrm{C}$. IR (KBr): v 3032 (br, $\mathrm{NH}), 1690$ (CO). ${ }^{1} \mathrm{H}$ NMR (300 MHz, DMSO-d $)$ : $\delta 1.26(\mathrm{br}, 4 \mathrm{H}, \mathrm{CH} 2), 3.05\left(\mathrm{br}, 4 \mathrm{H}, \mathrm{CH}_{2} \mathrm{~N}\right), 4.50\left(\mathrm{~s}, 4 \mathrm{H}, \mathrm{CH}_{2} \mathrm{O}\right)$, 5.15 (s, 2H, Pyridine -H), 6.70-7.10 ( m, 10H, Ar-H+NH), $8.35(b r, 2 H, N H), 10.72(b r, 4 H, N H), 11.15(b r, 4 H$, $\mathrm{NH}) .{ }^{13} \mathrm{C}$ NMR $(75 \mathrm{MHz}$, DMSO-d $)$ : 26.6, 37.8, 38.6, 67.0, 89.6, 110.9, 120.2, 121.0, 127.0, 129.4, 135.4, 143.8, 153.9, 162.5, 168.0. MS (El, $70 \mathrm{eV}): \mathrm{m} / \mathrm{z}(\%) 850\left[\mathrm{M}^{+}\right]$. Anal. Calcd for $\mathrm{C}_{38} \mathrm{H}_{3} 4 \mathrm{~N}_{12} \mathrm{O}_{12}: \mathrm{C}, 53.65 ; \mathrm{H}, 4.03 ; \mathrm{N}, 19.76$ found C, 53.84; H, 3.79; N, 20.02.

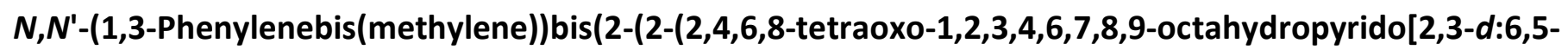

$\boldsymbol{d}^{\prime}$ ]dipyrimidin-5-yl)phenoxy)acetamide) (4d). Pale yellow powder (89\%). $\mathrm{mp}>300{ }^{\circ} \mathrm{C}$. IR (KBr): v $3201(\mathrm{NH})$, 3070 (br, NH), 1705 (CO). ${ }^{1} \mathrm{H}$ NMR (300 MHz, DMSO-d $): \delta 4.19-4.21\left(\mathrm{~d}, J=6.3 \mathrm{~Hz}, 4 \mathrm{H}, \mathrm{CH}_{2} \mathrm{~N}\right), 4.78\left(\mathrm{~s}, 4 \mathrm{H}, \mathrm{CH}_{2} \mathrm{O}\right)$, 
6.90-7.31 (m, 14H, Ar-H+NH), 7.76-7.78 (t, J = 6.3 Hz, 2H, NHCO), 11.15 (br, 4H, NH), 11.84 (br, 4H, NH). MS (EI, $70 \mathrm{eV}): \mathrm{m} / z(\%) 894\left[\mathrm{M}^{+}\right]$. Anal. Calcd for $\mathrm{C}_{42} \mathrm{H}_{30} \mathrm{~N}_{12} \mathrm{O}_{12}: \mathrm{C}, 56.38 ; \mathrm{H}, 3.38 ; \mathrm{N}, 18.78$ found C, 56.17; H, 3.56; N, 19.04.

\section{Supplementary Material}

Supplementary material related to this article, including Nuclear Magnetic Resonance $\left({ }^{1} \mathrm{H}\right.$ and ${ }^{13} \mathrm{C}$ NMR) figures for all new compounds $\mathbf{3 a}, \mathbf{3 c}, \mathbf{8}, \mathbf{4 a}$ and $\mathbf{4 d}$ are available in the online version of the text.

\section{References}

1. Shamim, T.; Gupta, M.; Paul, S. J. Mol. Catal. A Chem. 2009, 302, 15-19.

https://doi.org/10.1016/j.molcata.2008.11.024

2. Gómez-Pliego, R.; Gómez-Zamudio, J.; Velasco-Bejarano, B.; Ibarra-Barajas, M.; Villalobos-Molina, R. J. Pharmacol. Sci. 2013, 122, 184-192.

https://doi.org/10.1254/jphs.12248FP

3. Ibrahim, N.S.; Mohamed, M.F.; Elwahy, A.H.M.; Abdelhamid, I.A. Lett. Drug Des. Discov. 2018, 15, 10361045.

https://doi.org/10.2174/1570180815666180105162323

4. Sridhar, R.; Perumal, P.T. Tetrahedron 2005, 61, 2465-2470.

https://doi.org/10.1016/j.tet.2005.01.008

5. Paul, S.; Sharma, S.; Gupta, M.; Choudhary, D.; Gupta, R. Bull. Korean Chem. Soc. 2007, 28, 336-338. https://doi.org/10.5012/bkcs.2007.28.2.336

6. Saikh, F.; De, R.; Ghosh, S. Tetrahedron Lett. 2014, 55, 6171-6174.

https://doi.org/10.1016/j.tetlet.2014.09.025

7. Sridharan, V.; Perumal, P.T.; Avendaño, C.; Menéndez, J.C. Tetrahedron 2007, 63, 4407-4413.

https://doi.org/10.1016/i.tet.2007.03.092

8. Navidpour, L.; Shafaroodi, H.; Miri, R.; Dehpour, A.R.; Shafiee, A. Farmaco 2004, 59, 261-269. https://doi.org/10.1016/i.farmac.2003.11.013

9. Debache, A.; Ghalem, W.; Boulcina, R.; Belfaitah, A.; Rhouati, S.; Carboni, B. Tetrahedron Lett. 2009, 50, 5248-5250. https://doi.org/10.1016/j.tetlet.2009.07.018

10. Miri, R.; Javidnia, K.; Hemmateenejad, B.; Tabarzad, M.; Jafarpour, M. Chem. Biol. Drug Des. 2009, 73, 225-235.

https://doi.org/10.1111/j.1747-0285.2008.00770.x

11. Abbas, H.A.S.; El Sayed, W.A.; Fathy, N.M. Eur. J. Med. Chem. 2010, 45, 973-982.

https://doi.org/10.1016/j.ejmech.2009.11.039

12. Safak, C.; Simsek, R. Mini-Reviews Med. Chem. 2006, 6, 747-755.

https://doi.org/10.2174/138955706777698606

13. Murthy, Y.L.N.; Rajack, A.; Taraka Ramji, M.; Jeson Babu, J.; Praveen, C.; Aruna Lakshmi, K. Bioorganic Med. Chem. Lett. 2012, 22, 6016-6023.

https://doi.org/10.1016/i.bmcl.2012.05.003 
14. Samzadeh-Kermani, A.; Shafaroodi, H.; Miri, R.; Mirkhani, H.; Vosooghi, M.; Shafiee, A. Med. Chem. Res. 2009, 18, 112-126. c https://doi.org/10.1007/s00044-008-9112-5

15. Huber, I.; Wappl, E.; Herzog, A. Biochem. J. 2000, 836, 829-836. https://doi.org/10.1042/bj3470829

16. Ghozlan, S.A.S.; Ramadan, M.A.; Abdelmoniem, A.M.; Elwahy, A.H.M.; Abdelhamid, I.A. Turkish J. Chem. 2017, 41, $410-419$. https://doi.org/10.3906/kim-1609-42

17. Kassab, R.M.; Elwahy, A.H.M.; Abdelhamid, I.A. Monat. Chem. 2016, 147, 1227-1232. https://doi.org/10.1007/s00706-015-1644-z

18. Mohamed, M.F.; Darweesh, A.F.; Elwahy, A.H.M.; Abdelhamid, I.A. RSC Adv. 2016, 6, 40900-40910. https://doi.org/10.1039/c6ra04974e

19. Abdelhamid, I.A.; Darweesh, A.F.; Elwahy, A.H.M. Tetrahedron Lett. 2015, 56, 7085-7088. https://doi.org/10.1016/i.tetlet.2015.11.015

20. Fathalla, M.; Lawrence, C.M.; Zhang, N.; Sessler, J.L.; Jayawickramarajah, J. Chem. Soc. Rev. 2009, 38, 1608-1620.

https://doi.org/10.1039/b806484a

21. Sivakova, S.; Rowan, S.J. Chem. Soc. Rev. 2005, 34, 9-21. https://doi.org/10.1039/b304608g

22. Dinner, A.R.; Blackburn, G.M.; Karplus, M. Nature 2001, 413, 752-755. https://doi.org/10.1038/35099587

23. Di Noia, J.; Neuberger, M.S. Nature 2002, 419, 43-48. https://doi.org/10.1038/nature00981

24. Tucci, F.C.; Zhu, Y.F.; Guo, Z.; Gross, T.D.; Connors Jr., P.J.; Gao, Y.; Rowbottom, M.W.; Struthers, R.S.; Reinhart, G.J.; Xie, Q.; et al. J Med Chem 2004, 47, 3483-3486.

https://doi.org/10.1021/jm049791w

25. Sutherlin, D.P.; Sampath, D.; Berry, M.; Castanedo, G.; Chang, Z.; Chuckowree, I.; Dotson, J.; Folkes, A.; Friedman, L.; Goldsmith, R.; Heffron, T.; Lee, L.; Lesnick, J.; Lewis, C.; Mathieu, S.; Nonomiya, J.; Olivero, A.; Pang, J.; Prior, W. W.; Salphati, L.; Sideris, S.; Tian, Q.; Tsui, V.; Wan, N. C.; Wang, S.; Wiesmann, C.; Wong, S.; Zhu, B. Y. J. Med. Chem. 2010, 53, 1086-1097.

https://doi.org/10.1021/jm901284w

26. Brognara, E.; Lampronti, I.; Breveglieri, G.; Accetta, A.; Corradini, R.; Manicardi, A.; Borgatti, M.; Canella, A.; Multineddu, C.; Marchelli, R.; et al. Eur. J. Pharmacol. 2011, 672, 30-37. https://doi.org/10.1016/i.ejphar.2011.09.024

27. Liu, Y.Y.; Zeng, S.Y.; Leu, Y.L.; Tsai, T.Y. J. Agric. Food Chem. 2015, 63, 7333-7342. https://doi.org/10.1021/acs.jafc.5b01649

28. Tobe, M.; Isobe, Y.; Goto, Y.; Obara, F.; Tsuchiya, M.; Matsui, J.; Hirota, K.; Hayashi, H. Bioorganic Med. Chem. 2000, 8, 2037-2047. https://doi.org/10.1016/S0968-0896(00)00126-7

29. Sapozhnikova, K.A.; Slesarchuk, N.A.; Orlov, A.A.; Khvatov, E. V.; Radchenko, E. V.; Chistov, A.A.; Ustinov, A. V.; Palyulin, V.A.; Kozlovskaya, L.I.; Osolodkin, D.I.; et al. RSC Adv. 2019, 9, 26014-26023. https://doi.org/10.1039/c9ra06313g

30. Geant, P.Y.; Uttaro, J.P.; Périgaud, C.; Mathé, C. Molecules 2020, 25, 3708. https://doi.org/10.3390/molecules25163708 
31. Maslova, A.A.; Matyugina, E.S.; Snoeck, R.; Andrei, G.; Kochetkov, S.N.; Khandazhinskaya, A.L.; Novikov, M.S. Molecules 2020, 25. https://doi.org/10.3390/molecules25153350

32. Fatma, S.; Bishnoi, A.; Singh, V.; Al-Omary, F.A.M.; El-Emam, A.A.; Pathak, S.; Srivastava, R.; Prasad, O.; Sinha, L. J. Mol. Struct. 2016, 1110, 128-137. https://doi.org/10.1016/i.molstruc.2016.01.054

33. Suresh, T.; Nandha Kumar, R.; Mohan, P.S. Heterocycl. Commun. 2003, 9, 203-208. https://doi.org/10.1515/HC.2003.9.2.203

34. Maddila, S.; Naicker, K.; Gorle, S.; Rana, S.; Yalagala, K.; N. Maddila, S.; Singh, M.; Singh, P.; B. Jonnalagadda, S. Anticancer. Agents Med. Chem. 2016, 16, 1031-1037.

https://doi.org/10.2174/1871520616666151123095932

35. Neamati, N. Expert Opin. Investig. Drugs 2003, 12, 289-292. https://doi.org/10.1517/13543784.12.2.289

36. Pannecouque, C.; Pluymers, W.; Van Maele, B.; Tetz, V.; Cherepanov, P.; De Clercq, E.; Witvrouw, M.; Debyser, Z. Curr. Biol. 2002, 12, 1169-1177.

37. https://doi.org/10.1016/S0960-9822(02)00952-1 Elwahy, A.H.M.; Shaaban, M.R. Curr. Org. Synth. 2015, 11, 835-873. https://doi.org/10.2174/157017941106141023114039

38. Zhu, J.; Bienaymé, H. Multicomponent Reactions; Zhu, J., Bienaymé, H., Eds.; Wiley-VCH: Weinheim, Germany, 2005; ISBN 3527308067.

39. Elwahy, A.; Shaaban, M. Curr. Org. Synth. 2010, 7, 433-454. https://doi.org/10.2174/157017910792246117

40. Elwahy, A.H.M.; Shaaban, M.R. Curr. Org. Synth. 2015, 10, 425-466. https://doi.org/10.2174/1570179411310030007

41. Shaaban, M.R.; Elwahy, A.H.M. Curr. Org. Synth. 2015, 11, 471-525. https://doi.org/10.2174/15701794113106660076

42. Khoobi, M.; Ramazani, A.; Foroumadi, A.; Souldozi, A.; Ślepokura, K.; Lis, T.; Mahyari, A.; Shafiee, A.; Joo, S.W. Helv. Chim. Acta 2013, 96, 906-918.

https://doi.org/10.1002/hlca.201200187

43. Zareai, Z.; Khoobi, M.; Ramazani, A.; Foroumadi, A.; Souldozi, A.; Ślepokura, K.; Lis, T.; Shafiee, A. Tetrahedron 2012, 68, 6721-6726. https://doi.org/10.1016/j.tet.2012.05.112

44. Khoobi, M.; Ramazani, A.; Mahdavi, M.; Foroumadi, A.; Emami, S.; Joo, S.W.; Ślepokura, K.; Lis, T.; Shafiee, A. Helv. Chim. Acta 2014, 97, 847-853.

https://doi.org/10.1002/hlca.201300310

45. Ramazani, A.; Khoobi, M.; Torkaman, A.; Zeinali Nasrabadi, F.; Forootanfar, H.; Shakibaie, M.; Jafari, M.; Ameri, A.; Emami, S.; Faramarzi, M.A.; et al. Eur. J. Med. Chem. 2014, 78, 151-156. https://doi.org/10.1016/j.ejmech.2014.03.049

46. Sanad, S.M.H.; Kassab, R.M.; Abdelhamid, I.A.; Elwahy, A.H.M. Heterocycles 2016, 92, 910-924. https://doi.org/10.3987/COM-16-13441

47. Sharma, M.G.; Rajani, D.P.; Patel, H.M. R. Soc. Open Sci. 2017, 4, 170006. https://doi.org/10.1098/rsos.170006

48. Patel, D.M.; Patel, H.J.; Padrón, J.M.; Patel, H.M. RSC Adv. 2020, 10, 19600-19609. https://doi.org/10.1039/D0RA02990D 
49. Patel, D.M.; Sharma, M.G.; Vala, R.M.; Lagunes, I.; Puerta, A.; Padrón, J.M.; Rajani, D.P.; Patel, H.M. Bioorg. Chem. 2019, 86, 137-150.

https://doi.org/10.1016/i.bioorg.2019.01.029

50. Patel, D.M.; Patel, H.M. ACS Sustain. Chem. Eng. 2019, 7, 18667-18676.

https://doi.org/10.1021/acssuschemeng.9b05184

51. Santosh, R.; Paul, P.; Selvam, M.K.; Raril, C.; Krishna, P.M.; Manjunatha, J.G.; Nagaraja, G.K.

ChemistrySelect 2019, 4, 990-996.

https://doi.org/https://doi.org/10.1002/slct.201803416

52. Darwish, E.S.; Abdelhamid, I.A.; Nasra, M.A.; Abdel-Gallil, F.M.; Fleita, D.H. Helv. Chim. Acta 2010, 93, 1204-1208.

https://doi.org/10.1002/hlca.200900355

53. Abdelhamid, I.A.; Ghozlan, S.A.S.; Kolshorn, H.; Meier, H.; Elnagdi, M.H. Heterocycles 2007, 71, 26272637.

https://doi.org/10.3987/com-07-11141

54. Ghozlan, S.A.S.; Abdelhamid, I.A.; Gaber, H.M.; Elnagdi, M.H. J. Heterocycl. Chem. 2005, 42, $1185-1189$. https://doi.org/10.1002/jhet.5570420623

55. Ghozlan, S.A.S.; Ahmed, A.G.; Abdelhamid, I.A. J. Heterocycl. Chem. 2016, 53, 817-823. https://doi.org/10.1002/jhet.2341

56. Abdelhamid, I.A.; Darwish, E.S.; Nasra, M.A.; Abdel-Gallil, F.M.; Fleita, D.H. Synthesis (Stuttg). 2010, 11071112.

https://doi.org/10.1055/s-0029-1219235

57. Abdella, A.M.; Moatasim, Y.; Ali, M.A.; Elwahy, A.H.M.; Abdelhamid, I.A. J. Heterocycl. Chem. 2017, 54, 1854-1862.

https://doi.org/10.1002/jhet.2776

58. Abdelmoniem, A.M.; Ghozlan, S.A.S.; Abdelmoniem, D.M.; Elwahy, A.H.M.; Abdelhamid, I.A. J. Heterocycl. Chem. 2017, 54, 2844-2849.

https://doi.org/10.1002/jhet.2890

59. Abdelmoniem, A.M.; Salaheldin, T.A.; Abdelhamid, I.A.; Elwahy, A.H.M. J. Heterocycl. Chem. 2017, 54, 2670-2677.

https://doi.org/10.1002/jhet.2867

60. Darweesh, A.F.; Abd El-Fatah, N.A.; Abdelhamid, I.A.; Elwahy, A.H.M.; Salem, M.E. Synth. Commun. 2020, 50, 2531-2544.

https://doi.org/10.1080/00397911.2020.1784436

61. Eid, E.M.; Hassaneen, H.M.E.; Abdelhamid, I.A.; Elwahy, A.H.M. J. Heterocycl. Chem. 2020, 57, $2243-2255$. https://doi.org/10.1002/jhet.3945

62. Abdella, A.M.; Abdelmoniem, A.M.; Abdelhamid, I.A.; Elwahy, A.H.M. J. Heterocycl. Chem. 2020, 57, 14761523.

https://doi.org/10.1002/jhet.3883

63. Abdella, A.M.; Mohamed, M.F.; Mohamed, A.F.; Elwahy, A.H.M.; Abdelhamid, I.A. J. Heterocycl. Chem. 2018, 55, 498-507.

https://doi.org/10.1002/jhet.3072

64. Salama, S.K.; Mohamed, M.F.; Darweesh, A.F.; Elwahy, A.H.M.; Abdelhamid, I.A. Bioorg. Chem. 2017, 71, 19-29.

https://doi.org/10.1016/j.bioorg.2017.01.009 
65. Salama, S.K.; Darweesh, A.F.; Abdelhamid, I.A.; Elwahy, A.H.M. J. Heterocycl. Chem. 2017, 54, $305-312$. https://doi.org/10.1002/jhet.2584

66. Salem, M.E.; Darweesh, A.F.; Mekky, A.E.M.; Ahmad M. Farag, A.; Elwahy, and A.H.M. J. Heterocycl. Chem. 2017, 54, 226-234.

https://doi.org/10.1002/ihet.2571

67. El-Fatah, N.A.A.; Darweesh, A.F.; Mohamed, A.A.; Abdelhamid, I.A.; Elwahy, A.H.M. Monatshefte fur Chemie 2017, 148, 2107-2122. https://doi.org/10.1007/s00706-017-2040-7

68. Abd El-Fatah, N.A.; Darweesh, A.F.; Mohamed, A.A.; Abdelhamid, I.A.; Elwahy, A.H.M. Tetrahedron 2017, 73, 1436-1450.

https://doi.org/10.1016/j.tet.2017.01.047

69. Diab, H.M.; Abdelhamid, I.A.; Elwahy, A.H.M. Synlett 2018, 29, 1627-1633. https://doi.org/10.1055/s-0037-1609967

70. M. Abdella, A.; H. M. Elwahy, A.; A. Abdelhamid, I. Curr. Org. Synth. 2016, 13, 601-610. https://doi.org/10.2174/1570179413999151211115100

71. Al-Awadi, N.A.; Abdelhamid, I.A.; Al-Etaibi, A.M.; Elnagdi, M.H. Synlett 2007, 2205-2208. https://doi.org/10.1055/s-2007-985573

72. Abdelhamid, I.A.; Darwish, E.S.; Nasra, M.A.; Abdel-Gallil, F.M.; Fleita, D.H. Arkivoc 2008, 2008, $117-121$. https://doi.org/10.3998/ark.5550190.0009.h11

73. Al-Awadi, N.A.; Ibrahim, M.R.; Abdelhamid, I.A.; Elnagdi, M.H. Tetrahedron 2008, 64, 8202-8205. https://doi.org/10.1016/j.tet.2008.06.026

74. Abdella, A.M.; Abdelmoniem, A.M.; Ibrahim, N.S.; El-Hallouty, S.M.; Abdelhamid, I.A.; Elwahy, A.H.M. Mini-Reviews Med. Chem. 2019, 20, 801-816. https://doi.org/10.2174/1389557519666190919160019

75. Abdelmoniem, A.M.; Ghozlan, S.A.S.; Butenschön, H.; Abdelmoniem, D.M.; Elwahy, A.H.M.; Abdelhamid, I.A. Arkivoc 2019, 2019, 163-177.

https://doi.org/10.24820/ark.5550190.p010.875 